\title{
称理学 \\ ON THE VELOCITY-DISTRIBUTION AND THE MIXING LENGTH PECULIAR TO THE THIN SUPERCRITICAL FLOW STREAMING ON A ROUGH BED
}

\author{
By Dr. Eng., Shigeru Tanaka, C.E. Member* and Shüichi Sugimoto, C.E. Member**
}

Synopsis One of the characteristics of the thin supercritical flow running down a steep-slope pavement, a mountain-side, or a reservoir spillway, may be that the surface disturbances are remarkable. It may be considered that the disturbances are marked not only on the water-surface but also in the whole flow-profile. The research workers took up the uniform steady flow involving not only the said kind of flow but also channel and river flow, and studied especially how the mixing length of disturbances and velocity-distribution along the stream-profile varied with the flow-depth (measured normally to the bed surface), clarified the above relations theoretically, and finally induced two theoretical formulas. Then they performed many experiments concerning the velocity-distribution of the above titled flow, and determined the two constants involved in the theoretical formulas representing the mixing length relations, in order that the results obtained by the experiments and those obtained by using the above-mentioned two theoretical formulas might show good agreement. It is to be hoped that the writers also determine the two constants involved in the formula, so as to be able to apply the formulas induced by the writers to the river or channel flow, and then stress the characteristics of this kind of flow by comparing the values of these constants peculiar to the flow in question and an ordinary river or channel flow, if time permits. Up to now; they have been unable to ascertain these points, but they are intending to study further these details hereafter.

Introduction In the study of smooth pipe-flow, Prof. Prandtl was the first to introduce the concept of "the mixing length of turbulence" in the flow and clarified the characteristics of this flow. His way of thinking stood on the assumption that "the mixing length is proportionate to the flow depth". Then, V. Kármán ${ }^{1)}$ induced the mixing length by assuming the dynamical similarity of the flow. After that, H. Gebelein ${ }^{2)}$ made public the mixing length from his original way of thinking based. on the probability theory.

Meanwhile, concerning the turbulence of open channel-flow, many research workers tried to define the mixing length by applying the ways of thinking of the above mentioned authorities to this flow. Dr. Shōichirō Hayami ${ }^{3)}$ applied H. Gebelein's way of thinking to the open channel flow.

Lately, J. Rotta ${ }^{4}$ considered that the proportional relation between the mixing length and the flow depth would come into existence within the turbulent flow range over the laminar boundary layer, and calculated the velocity-distribution along the flow-profile in the case of the gentle-slope open channel flow. W. Szblewski ${ }^{5)}$ aquired the mixing length relations by the use of the similar assumption to that used by J. Rotta, for the convergent channel flow, and simultaneously proposed that the mixing length was to be concluded as the sum of two terms, i.e. the term which is proportionate to the first power of flow-depth and the one which is proportionate to the square of the 
same. Very recently, Yũichi Iwagaki ${ }^{6)}$ improved the idea of J. Rotta, and represented the relations between the mixing length and the flow-depth as a refracted line.

Thus although there are numerous important researches on the mixing length of the open channel flow, the fact is either there are still some points to be incomprehensible in the fundamental ideas of research workers or there are many researches based on the assumption that is far from the actual state, i.e. the relation between the mixing length and the flow-depth are supposed as nearly constant, irrespective of the flow-depth.

In this report, the writers induced theoretically the mixing length of turbulence in the open channel flow, setting up no such artificial assumptions beforehand which have been the foundations of the usual researches.

Besides, they are also introducing a fundamental equation by the use of which the velocitydistribution of the thin supercritical flow running on a rough steep bed is easily clarified; and this kind of equation has never been induced yet.

Fundamental Equations concerning Open Channel Flow Let us take a point on the bottomcenter-line of a straight open channel as an origin, and name the center-line running toward the lower stream-side along the channel-bottom-surface as " $x$ axis", and the line perpendicular to the " $x$ axis", running toward the water surface as " $y$ axis". Designate the velocity components referring to the above selected co-ordinate axes as " $u$ " and " $v$ ", respectively, the density of the flowing water as " $\rho$ ", the flow-depth along the " $y$ " axis as " $h$ ", the acceleration of gravity as " $g$ ", the inclined angle of the channel-bottom referring to the horizontal as " $\theta$ ", the pressure as " $p$ ", the viscositycoefficient of the flowing water as " $\mu$ ", the kinematic viscosity coefficient of the same as " $\nu$ "; then, considering the gravity force as the outer force, the Navier-Stokes equation can be expressed as follows :

$$
\rho\left(\frac{\partial u}{\partial t}+u \frac{\partial u}{\partial x}+v \frac{\partial u}{\partial y}\right)=\rho g \sin \theta-\frac{\partial p}{\partial x}+\frac{\partial}{\partial x}\left(\mu-\frac{\partial u}{\partial x}\right)+\frac{\partial}{\partial y}\left(\mu \frac{\partial u}{\partial y}\right) .
$$

If the flow is uniform and steady, we furthermore can assume $v=0, \mathrm{Eq}$. (1) is simplified and expressed as follows :

$$
\rho g \sin \theta+\frac{d}{d y}\left(\mu \frac{d u}{d y}\right)=0 .
$$

When turbulence exists in the flow, the shearing stress due to the turbulence, i.e. the Reynolds stress $\rho l^{2}\left|\frac{d u}{d y}\right| \frac{d u}{d y}$, should be taken into consideration, where " $l$ " is the mixing length of turbulence. Then, the following equation exists:

$$
\rho g \sin \theta+\frac{d}{d y}\left\{\left(\mu+\rho I^{2}\left|\frac{d u}{d y}\right|\right)-\frac{d u}{d y}\right\}=0 .
$$

Integrating this differential equation with respect to " $y$ ", we have:

$$
y \rho g \sin \theta+\rho\left(\nu+l^{2} \frac{d u}{d y}\right) \frac{d u}{d y}=\mathrm{const} \text {. }
$$

In this equation, using the condition that at the channel-bottom, i.e. $y=0, l=0$; we have:

$$
\left(\rho \nu \frac{d u}{d y}\right)_{y=0}=\left(\mu \frac{d^{\top} u}{d y}\right)_{y=0}=\text { const. }
$$

If we represent $\left(\mu \frac{d u}{d y}\right)_{y=0}=\tau_{0}$, then we get : 


$$
\text { const }=\tau_{0} \text {. }
$$

Then, Eq. (4) can be written in the form:

$$
g y \sin \theta+\left(\nu+l^{2} \frac{d u}{d y}\right) \frac{d u}{d y}=\frac{\tau_{0}}{\rho}=u^{* 2} .
$$

Now, this equation also be expressed as follows, by using the relation $\xi=y / h$ :

$$
\frac{l^{2}}{h^{2}}\left(\frac{d u}{d \xi}\right)^{2}+\frac{\nu}{h}\left(\frac{d u}{d \xi}\right)-\left(\frac{\tau_{0}}{\rho}-\xi g h \sin \theta\right)=0 .
$$

In the uniform-steady flow, the following equilibrium condition should be expressed as follows :

$$
\frac{\tau_{0}}{\rho}=g h \sin \theta
$$

Then, we get:

$$
\frac{l^{2}}{h^{2}}\left(\frac{d u}{d \xi}\right)^{2}+\frac{\nu}{h} \frac{d u}{d \xi}-g h \sin \theta(1-\xi)=0 .
$$

Therefore, we have:

$$
\frac{d u}{d \xi}=\frac{1}{2} \frac{1}{\left(\frac{l}{h}\right)^{2}}\left[\frac{\nu}{h}+\sqrt{\frac{\nu^{2}}{h^{2}}+\left(\frac{l}{h}\right)^{2}(1-\xi) 4 g h \sin \theta}\right] .
$$

If we put $\frac{\nu^{2}}{h^{2}} \fallingdotseq 0$, we get :

$$
\frac{d u}{d \xi}=\frac{1}{2} \frac{1}{\left(\frac{l}{h}\right)^{2}}\left[\frac{\nu}{h}+\frac{l}{h} \sqrt{1-\xi} \sqrt{4 g h \sin \theta}\right] .
$$

Given the suitable values to $l / h$, we are able to get the velocity-distribution ( $u$ versus $y$ ) of the flow, by integrating the Eq. (9') with respect to $\xi$ or $y$.

The Mixing Length In the first place, we ought to remind ourselves of the physical meaning of the mixing phenomena ${ }^{7}$ of turbulent flow.

When we consider the said mixing phenomena, it may be easily understood that some physical quantities are transported from one layer to another and the diffusion-phenomena of the quantities may happen, on the basis of the mixing of lines of flow.

This fact is, for example, just like in the following case, i.e. when fine particles take the Brownian movement; heat, momentum, etc. are transported from a layer to another together with the movement. Namely, some fluid-mass in a layer moves across the mean flow transversely, carrying some physical characteristics held in the original layer, and get to another one, and there the brought-in characteristics and the ones peculiar to the layer melt together, and thus the transportation and diffusion by turbulence-mixing take place.

Thus the distance where the physical quantities are transported across the mean flow transversely is called "the mixing length", corresponding to the so-called "mean free distance" in the case of molecular heat motion.

Accordingly, this distance $l$ is only the expected one obtained by means of statistics based on the mixing of flow lines, and the distance $l$ is a function of water-depth. This latter fact only is corresponding to the probability-distribution of the diffusion, namely the distribution of probability-density versus $y$. 
If we regard the diffusion-phenomena due to the turbulence of flow as a stochastic process and the transition-probability as making the so-called "Markoff's chain", the probability $f\left(x_{1}, x_{2}, x_{3}, t\right) d x_{1}$ $d x_{2} d x_{3}$ of which the state at the time $t$ exists in the extent between the co-ordinates $\left(x_{1}, x_{2}, x_{3}\right)$ and $\left(x_{1}+d x_{1}, x_{2}+d x_{2}, x_{3}+d x_{3}\right)$, was introduced by A.H. Kolmogoroff in the following equation:

$$
\frac{\partial f}{\partial t}+\sum_{k} \frac{\partial}{\partial x_{k}}\left(\alpha_{k} f\right)-\sum_{j} \sum_{k} \frac{\partial^{2}}{\partial x_{j} \partial x_{k}}\left(\beta_{j k} f\right)=0
$$

where $\alpha_{k}$ is a vector, and $\beta_{j k}$ is a variance tensor.

Now, when the open channel flow is uniform and steady, the mixing length $l$ becomes a function of $y$ only. Accordingly, if we now represent $l \propto f, f$ becomes a first order function of $y$, and Kolmogoroff's equation becomes as follows:

$$
\alpha \frac{d f}{d y}=\beta \frac{d^{2} f}{d y^{2}} .
$$

Integrating Eq. (11) with respect to $y$, we get

$$
\alpha f=\beta \frac{d f}{d y}+\gamma
$$

where $r$ is a integration's constant.

In case, the larger $y$ increases, the smaller dose the increase-rate of $f$ decreases to be, we put:

$$
\alpha / \beta=-\lambda \text {, }
$$

where $\lambda$ is a constant.

Then Eq. (12) becomes:

$$
\frac{d f}{d y}=-\lambda f+\frac{\gamma}{\beta} .
$$

When we integrate this differential equation with respect to $y$, we have:

$$
f=\frac{r}{\beta} \frac{1}{\lambda}+C e^{-\lambda y}
$$

where $C$ is an integration's constant.

If we use the conditions at $y=0, f=0$, we have:

$$
C=-\frac{\gamma}{\beta} \frac{1}{\lambda} \text {. }
$$

Eq. (14) becomes

$$
f=\frac{\gamma}{\beta} \frac{1}{\lambda}\left(1-e^{-\lambda y}\right) .
$$

Accordingly, as we take the mixing length $l$ to be in proportion to $f$, assuming $\kappa$ as constant, we get:

$$
\begin{aligned}
& l=\kappa\left(1-e^{-\lambda y}\right), \\
& \text { or } \quad l / h=A\left(1-e^{-B^{\xi}}\right), \quad \xi=y / h .
\end{aligned}
$$

If we interpret that at the free flow surface the fluid motion along the $y$ axis is not restricted, we are able to understand that the mixing length has some value even at the free flow surface.

Experimental Apparatuses and Method of Tests The experimental flume which we have been using is a wooden framed rectangular flume with glass pannels on both sides, the inside measurements of which are $20 \mathrm{~cm}$. wide, $15 \mathrm{~cm}$. high, and $734 \mathrm{~cm}$. long, and the bottom of which is roughened with the "Sōma-sand" grains fixed on the bottom plate (Fig. 1). Going into details, first the research 
workers painted the bottom plate with white paint, then sprinkled the "Somma sand" grains (the: average diameter being about $1 \mathrm{~mm}$.) over the undried paint-coating, and made the surface of sand. grains even with a trowel; and, after the paint was completely dried, they fixed the sand-grains on the flume-bottom by spraying transparent lacquer on them with a sprayer.

The uppermost portion of the flume is made to act as the receiver and simultaneously a flowregulator equipped with one gravel wall (the average dia. of gravel being $2.5 \mathrm{~mm}$.) sandwiched. between two $1 \mathrm{~mm}$.-mesh screens placed at $5 \mathrm{~cm}$. intervals and with another $1 \mathrm{~mm}$.-mesh screen placed in the lower stream side $20 \mathrm{~cm}$. apart from the lower screen of the gravel wall.

The elevated steel tank is $123 \mathrm{~cm}$. in inner diameter and $113.5 \mathrm{~cm}$. deep and is mounted on a trestle which is a timber frame work $330 \mathrm{~cm}$. high. Near the top edge of this tank an overflow pipe is equipped in order to keep the water level in the tank constant, and at the tank-bottom a $2.5^{\prime \prime}$ delivery pipe having a sluice valve is connected. Through the lower end of the pipe, water is poured into the uppermost portion of the flume.

The flowing water is circulated as follows: it is pumped up from a lower pit into the elevated tank, poured into the flume, flows down the flume, at the lower end of which the flow discharge is measured by the weighing apparatus receiving the water at a short period of time ( $5 \mathrm{sec} .-10 \mathrm{sec}$.), and the water falls into the return conduit leading the water to the pit.

The discharge is regulated by valve operation. The velocity meas-

Fig. 1 Diagrammatic arrangement of the experimental apparatuses urement is taken at the point in the lower stream side $495 \mathrm{~cm}$. apart from the lowest regulating screen. The total pressure is measured by means of the Pitot tube which is made of a stainless steel injector needle, 1.12 $\mathrm{mm}$. in outer diameter and $0.75 \mathrm{~mm}$. in inner diameter. The hydrostatic pressure is measured by the use of manometer connected to the hole 6.5

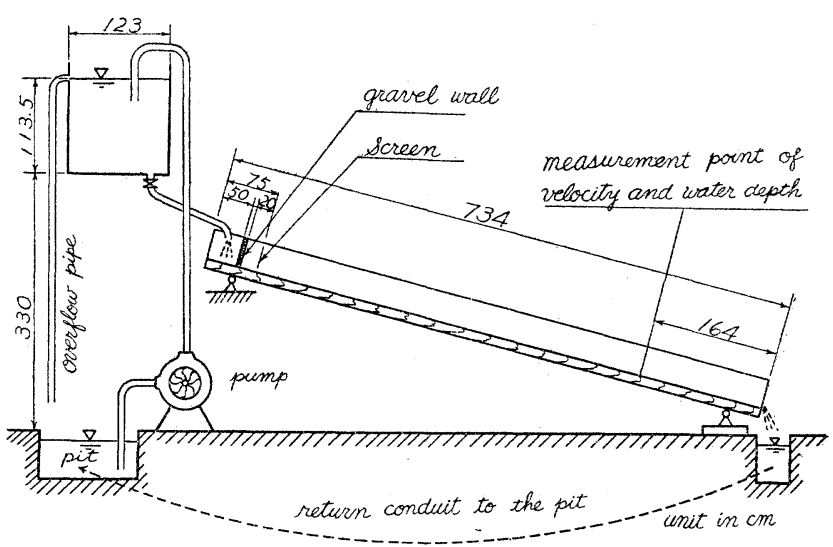
$\mathrm{mm}$. in inner diameter bored normally at the flume bottom, with a transparent vinyl pipe. The total pressure is measured at several points by the above-mentioned Pitot tube which is able to slide smoothly along the center line of the static pressure hole. The hydro-dynamic pressure is obtained by subtracting the hydro-static pressure from the total one.

The measurement of water-depth is rather difficult, owing to the fact that the surface-disturbances such as roll-waves and capillary-waves are rather predominant as compared with the thin flow-depth and the bottom unevenness.

We caught the lowest surface, and the crest of bottom-unevenness, by using the point gauge and watching carefully through the side glass plate. The summation of the difference of these two read. ings and the size of bottom unevenness is taken as the water depth. 
Experimental Procedure and Analysis of the Results Obtained We selected as the flume slopes, $1 / 10,1 / 7,1 / 5,1 / 4,1 / 3$, and as the mean discharges, $1000,1500,2000,3.000$ and 4000 cc./sec.. In each test we measured the velocities at some points on the center line of the statical head hole, from the bottom toward the water surface, and at each point the total and statical readings are taken three times precisely.

Now, we determine the values $A$ and $B$ in Eq. (16) by the trial and error method in the following way; firstly, assuming that $A$ and $B$ have proper values respectively, secondly, calculating the corresponding value of $l / h$, and thirdly, substituting this value for $l / h$ in Eq. $\left(9^{\prime}\right)$, we thus get the values of $u$ by integrating Eq. $\left(9^{\prime}\right)$ with respect to $\xi$ nummerically, (we use the nummerical calculation instead of pure integration because the latter is rather hard to use here), and compare the calculated $u$ values with the experimentally obtained ones to check if these values coincide with one another or not, and if they do not coincide, we repeat the above procedure until they become quite consistent.

The results thus obtained are shown in Fig. 2-6, and the results show good agreement with the experimental results, in consequence of the determination of proper values of $A$ and $B$. The values of $A$ and $B$ thus obtained are shown in Table 1 .

In this table, with regard to the values of $B$, in the lines of slopes, $1 / 10$,
Fig. 2 Curves representing the culculated velocity-distribution along the flowdepth, points showing the mean values of observed velocities taken three times precisely

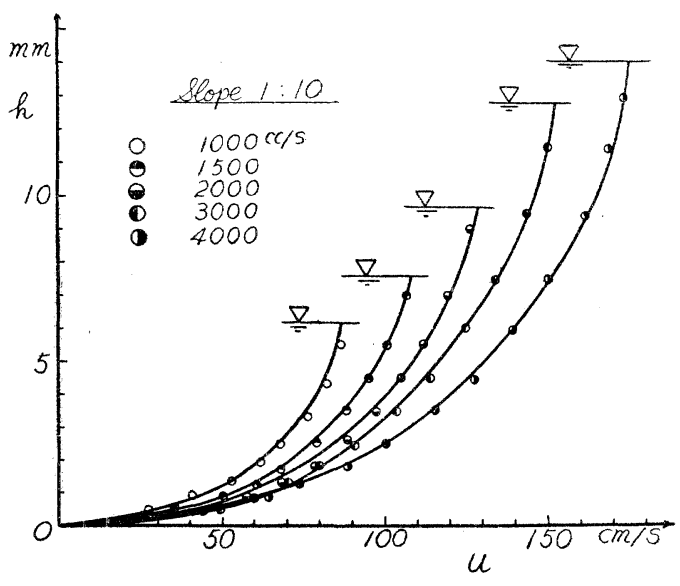

Fig. 3 Same the Fig. 2

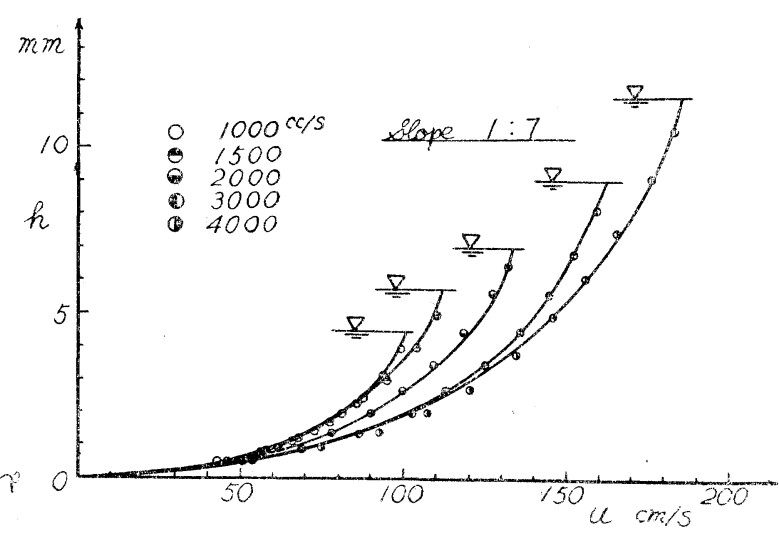

Fig. 4 Same the Fig. 2

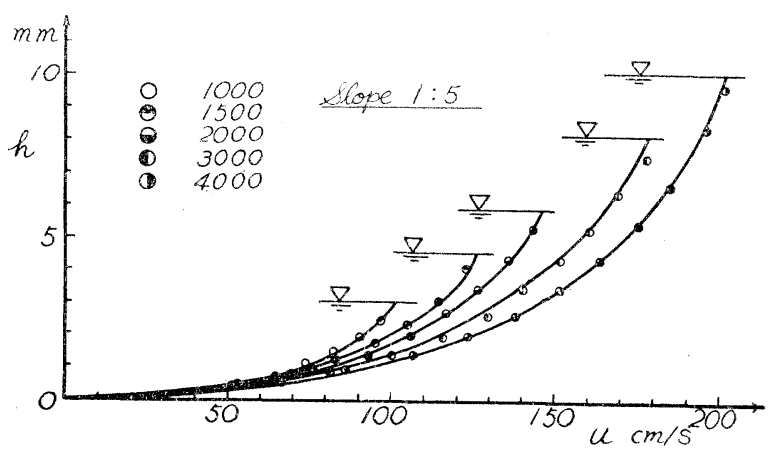
$1 / 7,1 / 5$, it is obvious that the majority of the values is 3 . So, in the case of $B=3$, if we take the $\sqrt{g h \sin \theta}$-value as the abscissa and the $A$-value as the ordinate, we are able to obtain a curve representing the relation between the values of $A$ and $\sqrt{g h \sin \theta}$ by plotting the $A$-value versus the $\sqrt{g h \sin \theta}-$ value, and the curve is shown in Fig. 7 . 
Table 1 The values of $A$ and $B$

\begin{tabular}{|c|c|c|c|c|c|c|c|c|c|c|}
\hline \multirow{3}{*}{$\frac{\text { Slope }}{1 / 10}$} & \multicolumn{5}{|c|}{$A$} & \multicolumn{5}{|c|}{$B$} \\
\hline & \multirow{2}{*}{$\begin{array}{l}1000 \\
0.150\end{array}$} & \multirow{2}{*}{$\begin{array}{l}1500 \\
0.132\end{array}$} & \multirow{2}{*}{$\begin{array}{r}2000 \\
0.125\end{array}$} & \multicolumn{2}{|c|}{$\begin{array}{c}\left.3000\right|^{4} 4000 \\
\mathrm{cc} / \mathrm{sec}\end{array}$} & \multirow{2}{*}{$\begin{array}{c}1000 \\
3\end{array}$} & \multirow{2}{*}{$\begin{array}{c}1500 \\
3\end{array}$} & \multirow{2}{*}{$\frac{2000}{3}$} & \multirow{2}{*}{$\begin{array}{r}3000 \\
\quad \mathrm{c} \\
3\end{array}$} & $\begin{array}{l}4000 \\
c\end{array}$ \\
\hline & & & & 0.120 & 0.108 & & & & & 3 \\
\hline $1 / 7$ & 0.136 & 0.136 & 0.125 & 0.115 & 0.118 & 3 & 3 & 3 & 3 & 3 \\
\hline $1 / 5$ & 0.136 & 0.128 & 0.125 & 0.118 & 0.160 & 3 & 3 & 3 & 3 & 2 \\
\hline $1 / 4$ & 0.098 & 0.125 & 0.118 & 0.160 & 0.150 & 4 & 3 & 3 & 2 & 2 \\
\hline $1 / 3$ & 0.100 & 0.104 & 0.112 & 0.157 & 0.153 & 5 & 4 & 3 & 2 & 2 \\
\hline
\end{tabular}

Fig. 5 Same the Fig. 2

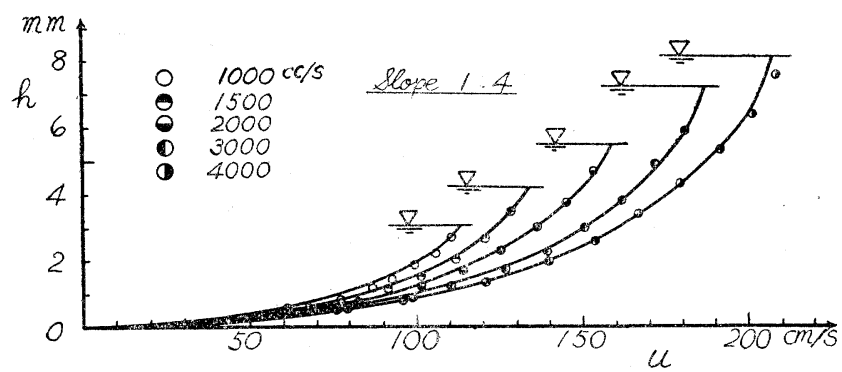

Fig. 6 Same the Fig. 2
Fig. 1 Curve representing $A$ value versus $\sqrt{g h \sin \theta}$-value

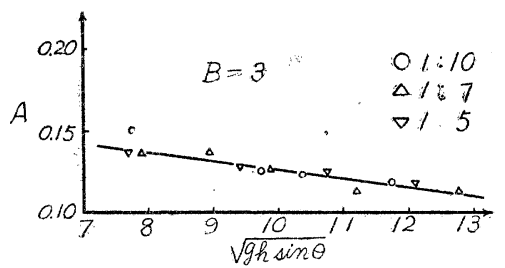

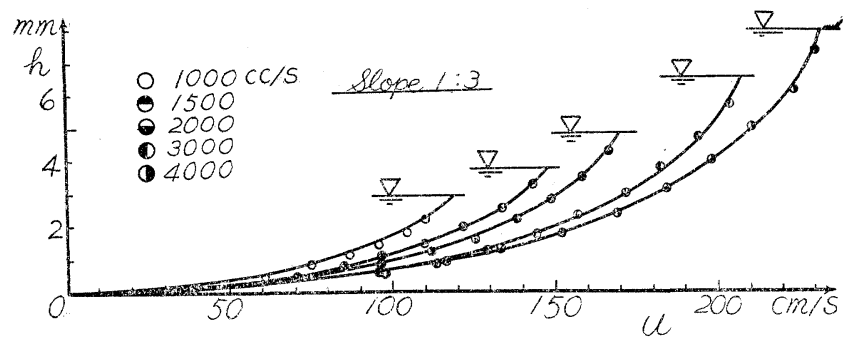

And, the calculated results of $l / h$ versus $y / h$ in each case by using the above obtained values of $A$ and $B$ are shown in Fig. 12 respectively.

Dr. S. Hayami formerly introduced the following formula showing the relation of the value of $l / h$ versus that of $l / y$ or $l / \xi$, applying the Gebelein's way of thinking to the open channel flow:

$$
l / h=C \frac{\xi(2-\xi)}{\sqrt{(2-\xi)^{2}+\xi^{2}}},
$$

where $C$ is a constant to be determined by the experimental results, and Dr. S. Hayami gave the following value based on the velccity-distribution obtained from the observation at the lower reaches

Fig. 8 Curves representing the calculated results of $l / h$ versus $y / h$

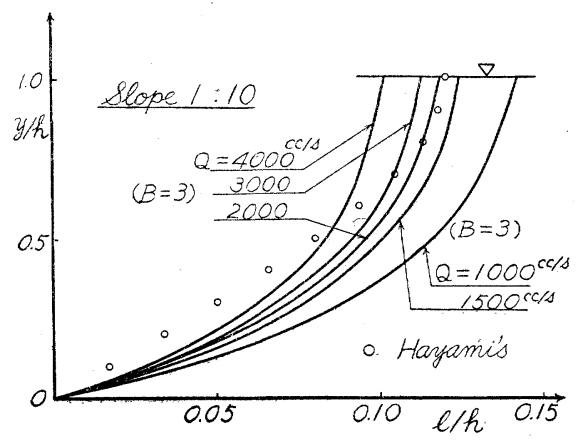

Fig. 9 Same the Fig. 8

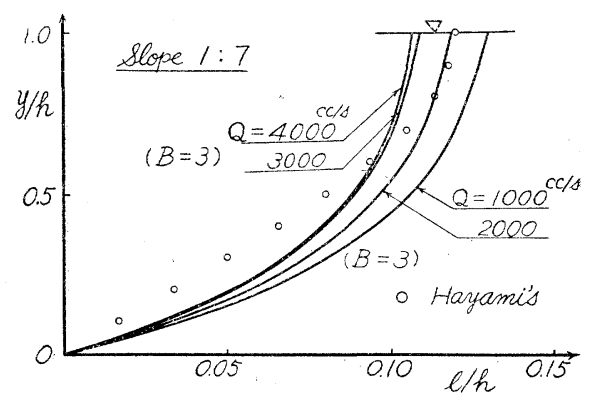


Fig. 10 Same the Fig. 8

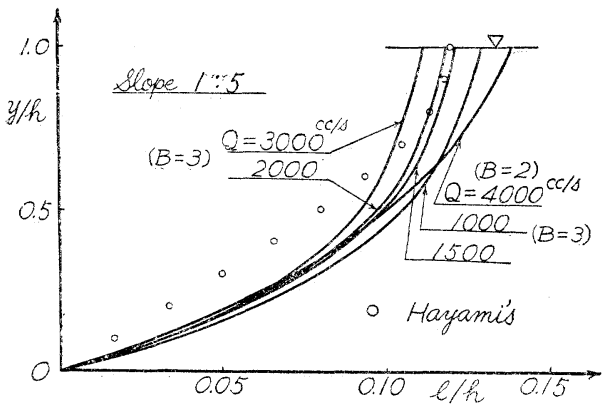

of the Yangtse-Kiang,

$$
C \doteqdot 0.17
$$

Calculating the values of $l / h$ in the cases of our tests by the use of this value of $C$, and plotting the values versus those of $y / h$, we have the points as marked $\circ$ in Fig. 8-12.

Referring to the curves in the abovementioned figures, it is certain that in the portion of bottom flow, the values obtained by using Hayami's formula are smaller as compared with those obtained by using Eq. (16), and on the surface the former values show approximately the average ones of the latter. Eq. (17) has been considered as the most reasonable one of the numerous formulas representing the value of $l / h$ in the open channel flow, and the fact that there exists the above-mentioned discrepancy is probably based on the disparity between our way of thinking and that of Gebelein.

Now, if we represent the mean flow-velocity as $u_{m}$, the size of the bottom roughness as $k$, and when we calculate the values of $h u_{m} / \nu, F=u_{m} / \sqrt{g h}$, and $k u^{*} / \nu$; we obtain the values which are shown in Table 2, based on our experimental data.

Table 2 The values of $h u_{m} / \nu, u_{m} / \sqrt{g h}$, and $k u^{*} / \nu$

\begin{tabular}{c||c|c|c|c}
\hline Slope & \multicolumn{2}{|c|}{$1: 10$} & \multicolumn{2}{|c}{$1: 3$} \\
\hline$Q \mathrm{cc} / \mathrm{sec}$ & 1000 & 4000 & 1000 & 4000 \\
\hline$h u_{\mathrm{m}} / \sqrt{g h}$ & 3130 & 13640 & 1820 & 10430 \\
\hline$F=u_{m} / \sqrt{g h}$ & 2.7 & 3.7 & 5.2 & 6.6 \\
\hline$k u^{*} / \nu$ & 58 & 122 & 68 & 112 \\
\hline
\end{tabular}

Conclusion In this paper the writers have introduced theoretically the mixing length of turbulence as a function of water-depth in the case of open channel flow, and simultaneously a new formula concerning the velocity-distribution of steep-slope-channel-flow with regard to which no reports of the theoretical researches have ever been made public so far. They have also determined the constants $A$ and $B$ involved in the mixing length equation by applying the experimental data to the velocitydistribution formula. The important item left for future study is to determine the values of $A$ and $B$ theoretically, at least either $A$ or $B$, and, if possible, botb $A$ and $B$. 
And yet, in order to give the bottom roughness, the writers have used only one kind of sand, i.e. the "Sōma-standard-sand", and it is preferable to use many other kinds of sand. If they had used many kinds of sand having different sizes to form bed unevenness, the constants $A$ and $B$ might show other different values from those shown in Table 1. If the values of $A$ and $B$ to be obtained from the use of many kinds of sand having different sizes were made clear through more tests, it might be of more help in applying the equations introduced by the writers to the technical aspects of this kind of flow. They much regret that they have had no available time allowance enough to perform tests in the instances of different conditions of bed-roughness in various sizes, and they are intending to continue such not-yet-conducted tests as early as possible.

Acknowledgement It is a pleasure to acknowledge instructive advices given this work by Dr. Eng. Tōjiro Ishihara and Yūichi Iwagaki in the University of Kyōto and the efforts put forth by Mr. Akira Kiyomoto.

\section{References}

(1) V. Kármán: Proc. 3 Int. Congr. App. Mech., Stockholm 1930, 1, 85-93.

(2) H. Gebelein : Türbulenz: Physikalische Statik und Hydrodynamik, Berlin, 1935.

(3) Shōichirō Hayami: Hydrological Studies on the Yangtze River, China; 4. On the mechanics of Flow in a Wide Alluvial River: The Journ. of the Shanghai Science Insti. Section 1, Vol. 1, July, 1939, 239-261.

(4) J. Rotta: Das in Wandnähe gültige Geschwindigkeitsgesetz türbulenter Strömungen, IngenieurArchiv, Band 18, 1950, 277-280.

(5) W. Szablewski : Türbulente Strömmungen in Konvergenten Kanälen, Ingenieur-Archiv, 20, 1952, $37-45$.

(6) Yuichi Iwagaki: On the Laws of Resistance to Turbulent Flow in Open Smooth Channels, Trans. J.S.C.E. Vol. 16, April 1953, 22-28.

(7) Goldstein: Modern Developments in Fluid Dynamics, Vol. 1, 1938, 205. 